\title{
ALGUNS ASPECTOS DO COMPORTAMENTO DE CEPAS SILVESTRES DE TRYPANOSOMA CRUZI EM CAMUNDONGOS E CALOMYS CALLOSUS (RODENTIA)*
}

Dalva A. Mello*:

Edna Valin ***

Maria Lucia Teixeira*:

\begin{tabular}{l|l|} 
RSPUB9/475 \\
\hline
\end{tabular}

MEllo, D. A. et al. Alguns aspectos do comportamento de cepas silvestres de Trypanosoma cruzi em camundongos e Calomys callosus (Rodentia). Rev. Saúde públ., S. Paulo, 13:314-25, 1979.

Resumo: Foram estudadas quatro cepas silvestres de T. cruzi: M226 isolada de Calomys callosus (Rodentia) e R52, R64 e R65 isoladas de Didelphis albiventris (Marsupialia). Estes animais foram coletados no municipio de Formosa, Goiás, Brasil. Os aspectos abordados, relacionados com o comportamento destas cepas em camundongos "swiss" 40 e C. callosus nascidos em laboratório, foram: parasitemia, prepatência, letalidade e histopatologia. Os resultados indicaram que as quatro cepas tinham baixa virulência para os animais testados. $A$ parasitemia sempre se apresentou baixa e regular para os C. callosus. Nos camandongos, só raramente a parasitemia era patente. A prepatência nos C. callosus variou em média entre 9,2 a 10,2 dias, enquanto nos camundongos ficou entre 12-48 dias. A letalidade para C. callosus foi $7,7 \%$ e para camundongos 12,0\%. Os estudos histopatológicos mostraram que somente 17,2\% dos C. callosus apresentaram parasitismo tissular, enquanto em $25 \%$ dos camundongos foram encontrados pseudocistos integros ou rompidos. Houve um nitido miotropismo das quatro cepas tanto nos camundongos quanto nos $\mathrm{C}$. callosus.

Unitermos: Trypanosoma cruzi. Animais de laboratório.

\section{N T RODUG $\mathrm{A} O$}

Durante estudos ecológicos sobre reservatórios de Trypanosoma cruzi em regiões do cerrado do Brasil Central, foram isoladas três cepas deste parasito de Didelphis albiventris (= azarae) (Marsupialia) e uma de Calomys callosus (Rodentia).

Sem dúvida, além da identificação especifica de amostras de $T$. cruzi isoladas de animais silvestres, o estudo destes parasitos "in vivo" ou "in vitro" pode contribuir com dados importantes à compreensão da manutenção do ciclo silvestre e suas implicaçōes epidemiológicas e clinicas. Neste último aspecto, Andrade 3 (1976) chama atenção para o fato de que o quadro clínico da

\footnotetext{
Trabalho realizado com auxilio do Conselho Nacional do Desenvolvimento Cientifico e Tecnológico (CNPq - PDE/02-1-06, FUB-CNPq. cadastro no 81.961).

: Do Departamento de Medicina Complementar da Faculdate de Ciencias da Saude da Universidale de Brasília - 70910 - Brasilia, DF - Brasil.

$* *$ Bolsista do CNPq.
} 
MELLO, D. A. et al. Alguns aspectos do comportamento de cepas silvestres de Trypanosoma cruzi em camundongos e Calomys callosus (Rodentia). Rev. Saúde públ., S. Paulo, 13:314-25, 1979 .

doença de Chagas pode estar relacionada com a cepa do $T$. cruzi.

Embora tenham sido realizadas várias observações experimentais sobre amostras de $T$. cruzi isoladas de marsupiais e roedores, muita coisa deixa a questionar. O presente trabalho tem por objetivo principal o estudo de alguns aspectos de comportamento "in vivo" das cepas silvestres mencionadas no primeiro parágrafo.

\section{MATERIAL E METODOS}

Origem das cepas de $\mathrm{T}$. cruzi

Foram estudadas quatro cepas de $T$. crli$z i$, isoladas de animais silvestres a saber: $R 52$ e $R 65$ isoladas de duas fêmeas de $D$. albiventris (Marsupialia), capturadas na mata do Abadio, região do Rio Canabrava, nordeste de Formosa, Goiás, respectivamente em 20 de agosto e 17 de setembro de 1975; R64, foi também isolada de uma fèmea de $D$. albiventris, capturada na mata do córrego da Picada, municipio de Mambaí, Goiás, em 2 de setembro de 1975; M226, isolada de uma fêmea de $C$. callosis (Rodentia), capturada em 10 de novembro de 1975 , na mesma região que as duas primeiras acima mencionadas. As quatro cepas apresentaram formas sanguícolas predominantemente largas.

As cepas foram isoladas a partir de xenos com ninfas do III, IV e $V$ estágios de Triatoma infestans, Triatoma sordida, Rhodnius neglectus e Panstrongylus megistus. A manutenção das cepas antes de serem iniciados os estudos experimentais, era feita em $C$. callosus nascidos em laboratório e camundongos albinos, através de inoculações de fezes de barbeiros positivos e xenos.

Estudos Experimentais "in vivo" com as cepas silvestres de $\mathrm{T}$. cruzi

Estes estudos foram iniciados um ano após as capturas dos animais infectados naturalmente com o $T$, cruzi.
Para experiências "in vivo" foram utilizadas duas espécies de roedores: $C$. callosus, com 34-48 dias de idade, procedentes de uma colônia mantida em laboratório a partir de setembro de 1974 e Mus musculus (camundongos) albino cepa "swiss" $40 \mathrm{com}$ 21-36 dias de idade. Para cada cepa os animais foram inoculados com fezes de barbeiros positivos com predominância de tripomastigotas, na proporção de $50 \%$ do material fecal com $50 \%$ de salina, o inóculo foi de $0,1 \mathrm{ml}$ por via intraperitoneal. $O$ número total de animais utilizados por cepa foi: $R 52-15$ C. callosus e 20 camundongos, $R 64-15$ C. callosus e 13 camundongos, R65 - 20 C. callosus e 20 camundongos e $M 226-15$ C. callosus e 10 camundongos.

Após os 4 dias que se seguiram as inoculaçōes, foram iniciados exames diários de sangue dos animais infectados, para observar o período de prepatência. Após a primeira deteç̧ão de tripomastigotas no sangue, os exames passavam a ser realizados em dias alternados com a contagem dos parasitos para seguir a evolução da parasitemia. A contagem dos parasitos foi feita de acordo com o método de Brener ${ }^{9}$ (1961). A parasitemia foi acompanhada durante 63 dias após às inoculações.

O período de manutenção dos animais inoculados que sobreviviam à infecção foi de 90 dias, quando então eram todos submetidos a xeno com ninfas de III, IV e $\mathrm{V}$ estágios de $T$. infestans, $R$. neglectus e $P$. megistus. Os triatomíneos assim alimentados eram examinados após 30 dias do repasto sanguíneo. Após os xenos eram sacrificados, e fragmentos do coração, fígado, baço, esôfago, intestino grosso e músculo da coxa eram fixados em formol $10 \%$ para os estudos histopatológicos. Os tecidos assim fixados e incluídos em parafina eram coletados em secçōes de $5 \mu \mathrm{m}$ de espessura e corados com hematoxilina-eosina. 
MELlo, D. A. et al. Alguns aspectos do comportamento de cepas silvestres de Trypanosoma cruzi em camundongos e Calomys callosus (Rodentia). Rev, Saúde públ., S. Paulo, 13:314-25. 1979.

\section{RESULTADOS}

As Tabelas 1 e 2 apresentam respectivamente os resultados obtidos sobre o periodo, de prepatência em $C$. callosus e camundongos infectados com as quatro cepas de $T$. cruzi.
$\mathrm{Na}$ Tabela 3 encontra-se a evolução da parasitemia em $C$. callosus acompanhada durante 63 dias após as inoculações. Como pode ser observado, a parasitemia foi relativamente baixa com a elevação gradual da curva. Os picos mais elevados situam-se entre o 23?-33n dia para a cepa $R 52,17$ ?-

T A B E L A 1

Período de prepatência em $C$. callosus infectados experimentalmente com cepas silvestres de $T$. cruzi.

\begin{tabular}{|c|c|c|c|c|}
\hline \multirow{2}{*}{$\begin{array}{l}\text { Cepas de } \\
T . \text { cruzi }\end{array}$} & \multicolumn{4}{|c|}{ Periodo de Prepatência (dias) } \\
\hline & \multicolumn{2}{|r|}{ Média } & \multicolumn{2}{|c|}{ Amplitude } \\
\hline$R 52$ & 9.20 & $( \pm 1.69)$ & 7 & 12 \\
\hline R64 & 10.20 & $( \pm 1,23)$ & 8 & 11 \\
\hline R65 & 9.30 & $( \pm 2.16)$ & 9 & 15 \\
\hline M226 & 9.80 & $( \pm 2,45)$ & 6 & 17 \\
\hline
\end{tabular}

T A B E L 2

Periodo de prepatência em camundongos albinos infectados com cepas silvestres de $T$. cruzi.

\begin{tabular}{|c|c|c|c|c|c|c|}
\hline $\begin{array}{l}\text { Cepas de } \\
T . \text { cruzi }\end{array}$ & & $\begin{array}{c}\text { No } \\
\text { Periodo }\end{array}$ & $\begin{array}{l}\text { de anima } \\
\text { prepatent }\end{array}$ & $\begin{array}{ll}\text { ais } / \\
\text { ite }\end{array}$ & as) & \\
\hline R52 & $1 / 12$ & $1 / 14$ & $1 / 16$ & $4 / 17$ & e & $1 / 35$ \\
\hline R64 & $1 / 14$ & $1 / 15$ & $1 / 17$ e & $1 / 29$ & & \\
\hline R65 & $1 / 26$ & e $1 / 48$ & & & & \\
\hline M226 & $1 / 45$ & & & & & \\
\hline
\end{tabular}

$31^{\circ}$ dia para a cepa M226. Nas quatro cepas estudadas, foi observado que a partir do 39 . dia após as inoculações a parasitemia decrescia. Apesar das diferenças nos dias dos picos mais elevados, pode ser observado, no entanto, uma nítida superposição na ampiltude observada, como mostram os dados da Tabela 3.

A evolução da parasitemia nos camundongos inoculados só foi possível ser acom- panhada em dois animais inoculados com a cepa $R 52$ e dois inoculados com a cepa R64 (Tabela 4). Os outros camundongos só raramente apresentaram parasitemia patente, sendo esta sempre em níveis muito baixos, variando entre 50 a 100 tripanosomas $/ 5 \mathrm{~mm}^{3}$.

Os dados apresentados sobre a letalidade nas duas espécies de animais infectados com as cepas silvestres de $T$. cruzi encon- 
MELLO, D. A. et al, Alguns aspectos do comportamento de cepas silvestres de Trypanosoma cruzi em camundongos e Calomys callosus (Rodentia). Rev. Saúde públ., S. Paulo, 13:314-25, 1979 .

T A B E L A 3

Evolução da parasitemia em C. callosus infectados experimentalmente com cepas silvestres de $T$. cruzi.

\begin{tabular}{|c|c|c|c|c|c|}
\hline \multirow{2}{*}{$\begin{array}{c}N^{\circ} \\
\text { após }\end{array}$} & \multirow{2}{*}{$\begin{array}{l}\text { de dias } \\
\text { inoculaçăo }\end{array}$} & \multicolumn{4}{|c|}{ Cepas de $T$. cruzi } \\
\hline & & $\mathrm{R} 52$ & R64 & R65 & M226 \\
\hline & 9 & 33.3 & 0 & 10 & 80 \\
\hline & 11 & 75 & 85 & 10 & 100 \\
\hline & 13 & 210 & 200 & 220 & 100 \\
\hline & 15 & 120 & 285 & 285 & 90 \\
\hline & 17 & 170 & 1.225 & 265 & 20 \\
\hline & 19 & 265 & 840 & 285,7 & $\therefore 0$ \\
\hline & 21 & 555 & 575 & 122,2 & 130 \\
\hline & 23 & 1.611 & 975 & 100 & 190 \\
\hline & 25 & 3.150 & 640 & 112.5 & 530 \\
\hline & 27 & 2.500 & 895 & 275 & 470 \\
\hline & 29 & 3.050 & 590 & 268.7 & 350 \\
\hline & 31 & 7.632 & 925 & 712,5 & 100 \\
\hline & 33 & 1.440 & 585 & 537.5 & 260 \\
\hline & 35 & 775 & 460 & 581,2 & 180 \\
\hline & 37 & 322,2 & 522 & 1.450 & 330 \\
\hline & 39 & 144,4 & 505 & 842,8 & 350 \\
\hline & 41 & 33,3 & 371 & 187.5 & 250 \\
\hline & 43 & 66,6 & 405 & 158.3 & 190 \\
\hline & 45 & 66.6 & 450 & 141,7 & 120 \\
\hline & 47 & 34 & 345 & 85.5 & 120 \\
\hline & 49 & 27,7 & 275 & 16,6 & 20 \\
\hline & 51 & 66.6 & 935,5 & 33.3 & 150 \\
\hline & 53 & 42 & 315 & 200 & 150 \\
\hline & 55 & 8.3 & 207.1 & 191.6 & 150 \\
\hline & 57 & 0 & 288,8 & 170 & 120 \\
\hline & 59 & 16.6 & 255 & 400 & 250 \\
\hline & 61 & 12,5 & 220 & 670 & 110 \\
\hline & 63 & 0 & 245 & 410 & 100 \\
\hline
\end{tabular}

tram-se registrados na Tabela 5. A letalidade no total de animais estudados foi significantemente maior para os camundongos $(12,0 \%)$ do que para os $C$. callosus $(7,7 \%)$. Considerando, entretanto, cada cepa isolada, a letalidade só foi significantemente maior para os camundongos inoculados com a R52 $(P<0,01)$.

Os resultados dos xenos aplicados em todos os animais inoculados foram positivos para $T$. cruzi, tendo sido encontradas formas epimastigotas e tripomastigotas.

Na Tabela 6 estão os dados sobre o numero de $C$. callosus e camundongos encontrados com parasitismo tecidual para as quatro cepas silvestres de $T$. cruzi estudadas. No total de 29 C. callosus estudados só foi possivel detectar pseudocistos contendo formas amastigotas em 5 indivíduos, i.e. em $17,2 \%$. Entre os camundongos, só em $25 \%$ (9 animais) foram encontrados 
MELLO, D. A. et al. Alguns aspectos do comportamento de cepas silvestres de Trypanosoma cruzi em camundongos e Calomys callosus (Rodentia). Rev, Saúde públ., S. Paulo. 13:314-25, 1979.

T A B E L A 4

Evolução da parasitemia em quatro camundongos inoculados com duas cepas silvestres de $T$, cruzi.

\begin{tabular}{|c|c|c|c|c|}
\hline $\begin{array}{l}\text { No de dias após } \\
\text { inoculaçăo (Parasi- } \\
\text { temia } / 5 \mathrm{~mm} 3 \text { ) }\end{array}$ & R52 & $\begin{array}{l}\text { Cepa } \\
\text { R52 }\end{array}$ & $\begin{array}{l}\text { cruzi } \\
\qquad \text { R64 }\end{array}$ & $\mathrm{R} 64$ \\
\hline 15 & 50 & 50 & 0 & 50 \\
\hline 17 & 200 & 100 & 0 & 0 \\
\hline 19 & 100 & 50 & 0 & 50 \\
\hline 21 & 100 & 250 & 200 & 0 \\
\hline 23 & 50 & 300 & 250 & 0 \\
\hline 25 & 0 & 200 & 750 & 0 \\
\hline 27 & 150 & 100 & 3.400 & 40 \\
\hline 29 & 250 & 50 & 2.300 & 750 \\
\hline 31 & 0 & 100 & 2.250 & 650 \\
\hline 33 & 150 & 50 & 650 & 4.750 \\
\hline 35 & 100 & 50 & 750 & 3.350 \\
\hline 37 & 0 & 0 & 500 & 2.300 \\
\hline 39 & 50 & 50 & 50 & 750 \\
\hline 41 & 50 & 0 & 0 & 550 \\
\hline $4: 3$ & 50 & 0 & 0 & 250 \\
\hline 45 & 0 & 0 & 0 & 550 \\
\hline 47 & 0 & 0 & 0 & 300 \\
\hline 49 & 50 & 0 & 0 & 150 \\
\hline 51 & 0 & 0 & 0 & 100 \\
\hline 53 & 50 & 0 & 50 & 0 \\
\hline 55 & 0 & 0 & 0 & 0 \\
\hline 57 & 0 & 0 & 0 & 0 \\
\hline 59 & 0 & 0 & 0 & 0 \\
\hline 61 & 0 & 0 & 0 & 0 \\
\hline
\end{tabular}

ninhos de amastigotas. Nas duas espécies de roedores o parasitismo tecidual encontrava-se na musculatura cardíaca e esquelética.

A Tabela 7 apresenta os resultados sobre o tropismo tecidual das quatro cepas de $T$. cruzi estudadas nas duas espécies de roedores experimentalmente infectados. Entre os C. callosus infectados e estudados do ponto de vista histopatológico, somente em
17 animais foram encontradas lesões com ou sem parasitismo tecidual. Entre os camundongos, somente 6 foram negativos para qualquer tipo de lesão ou reação. O quadro histopatológico tanto para o $C$. callosus quanto para o camundongo revelou reações inflamatórias, com ou sem parasito, consistindo de infiltrações de células mononucleares e destruição de miofibras e de células neuronais do plexo nervoso mioentérico 
MELLO, D. A. et al. Alguns aspectos do comportamento de cepas silvestres de Trypanosoma cruzi em camundongos e Calomys callosus (Rodentia). Rev. Saúde públ., S. Paulo, 13:314-25, 1979.

TA B E L A 5

Letalidade em $C$. callosus e $M$. musculus albino infectáos em laboratório com cepas silvestres de $T$. cruzi.

\begin{tabular}{|c|c|c|c|c|c|c|c|c|}
\hline \multirow{3}{*}{$\begin{array}{l}\text { Cepas de } \\
T . \quad \text { cruzi } \\
\text { R52 }\end{array}$} & \multicolumn{8}{|c|}{ Letalidade } \\
\hline & \multicolumn{3}{|c|}{$\begin{array}{r}\text { C. callosus } \\
\text { Inaculados/Mortos }\end{array}$} & \multirow{2}{*}{$\frac{\%}{6,6}$} & \multicolumn{4}{|c|}{$\begin{array}{l}\text { M. musculus albino } \\
\text { Inoculados/Mortos } \%\end{array}$} \\
\hline & 15 & / & 1 & & 15 & f & 3 & 20,6 \\
\hline R64 & 15 & 1 & 0 & 0.0 & 13 & 1 & 0 & 0,0 \\
\hline R65 & 20 & 1 & 4 & 20,0 & 20 & / & 2 & 10.0 \\
\hline M226 & 15 & 1 & 0 & 0,0 & 10 & / & 2 & 20,0 \\
\hline Total & 65 & / & 5 & 7,7 & 58 & / & 7 & 12,0 \\
\hline
\end{tabular}

T A B E L A 6

Número de animais, inoculados experimentalmente com cepas silvestres de $T$. cruzi, com parasitismo tecidual.

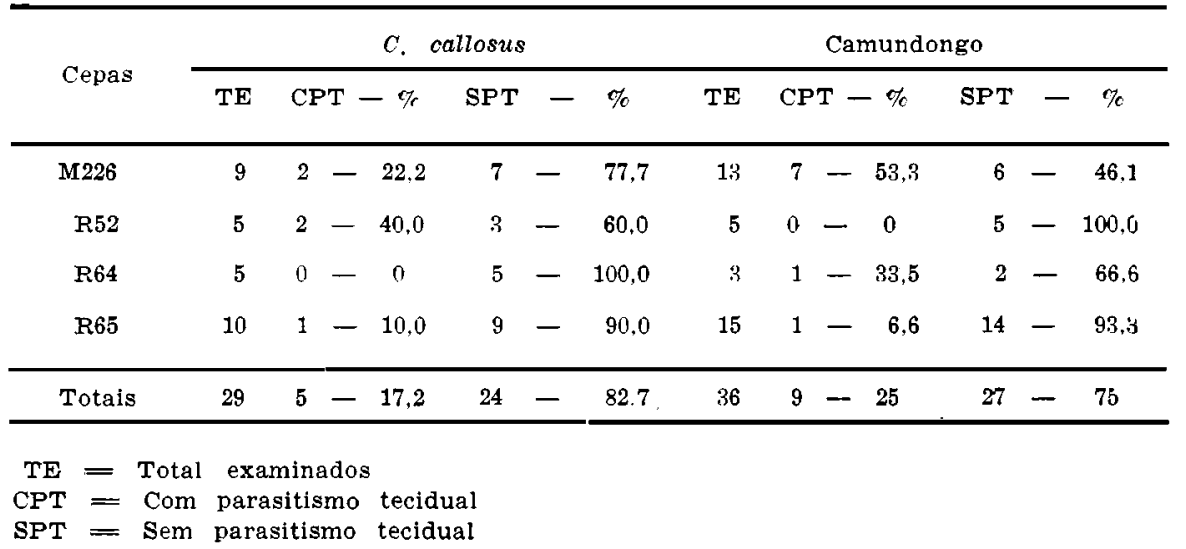

de tubo digestivo. Areas de fibrose foram também observadas em $C$. callosus e camundongos.

\section{COMENTARIOS E CONCLUSÕES}

Tem-se demonstrado que existem diferenças morfológicas e biológicas de cepas de $T$. cruzi isoladas de humanos e animais silvestres.
Brener e Chiari 10 (1963), Brener i.s (1965 e 1969), Andrade 2,3 (1974 e 1976) combinaram as variações morfológicas que ocorrem nas formas sanguícolas (largas, bojudas, delgadas) com o comportamento em meios acelulares e em camundongos. $O$ último autor verificou uma nitida relação entre essas diferentes formas de tripomasm tigotas, a evolução da infecção em camundongos e as manifestações clinicas no homem. Mais recentemente Sogayar ${ }^{82}$ (1978) 
MELLo, D. A. et al. Alguns aspectos do comportamento de cepas silvestres de Trypanosoma cruzi em camundongos e Calomys callosus (Rodentia). Rev. Saúde públ., S. Paulo, 13:314-25, 1979.

\section{T A B E L A 7}

Tropisno tecidual das cepas silvestres de $T$. cruzi em camundongos albinos e $C$. callosus infectados experimentalmente.

\begin{tabular}{lccccc}
\hline \multirow{2}{*}{ Cepas } & \multicolumn{2}{c}{ Tecidos parasitados e lesados } & \multicolumn{2}{c}{ Tecidos lesados } \\
\cline { 2 - 5 } & C. callosus & Camundongos & C. callosus & Camundongos \\
M226 & ME & ME & MIOC. & MIOC.ME.GMIOE. \\
R52 & ME & - & MIOC.ME.GMIOE. & MIOC.ME. \\
R65 & MIOC. & ME & MIOC.ME. & MIOC.ME. \\
\hline
\end{tabular}

ME. = Músculo Esquelético

MIOC. = Miocardio

GMIOE. = Gânglios mioentéricos

estudon o comportamento de 6 cepas de $T$. cruzi de humanos e de triatomíneos, en ratos, verificando resultados contraditórios aos então achados en camundongos. Todos esses trabalhos citados referem-se apenas a $T$. cruzi isolado de humanos ou triatomineos.

Nussenzweig e col.:- (1963) ao estudaren aspectos imunológicos de amostras isoladas de $D$. albiventris e $D$. marsupialis, verificaram comportamento diferente com relação à amostras humanas. Nussenzweig e Goble 20 (1966) estudando amostras de $T$. cruzi de diferentes origens mostraram que elas poderiam ser agrupadas em três tipos imunológicos distintos: tipo A - 5 cepas humanas, 2 de morcegos e 1 de triatomíneo; tipo B - 3 de gambá, 2 de macacos e 1 de roedor; e tipo $C-1$ de mustelídeo.

Miles e col:25 (1977) estudando isoenzimas, verificaram dois grupos padrões distintos de cepas de $T$. cruzi. Estes autores mostraram que este aspecto poderia ter importância epidemiológica, uma vez que a origem das amostras estudadas e agrupadas, no grupo I, haviam sido isoladas de ambiente doméstico, enquanto do grupo II, haviam sido isoladas do ambiente silvestre

Várias espécies de roedores e marsupiais têm sido incriminadas como reservatórios de
T. iruzi A literatura indica yue entre os primeiros, 4 espécies foram encontradas alberbergando este parasito. Entre os marsupiais conta-se até o momento com 14 espécies incriminadas como reservatórios do T. cruzi.

No Brasil, citam-se entre outros, os trábalhos de Deane 12 (1960), Ferriolli Filho e Barretto ${ }^{14.15 .16}$ (1965 e 1966), Siqueira e col.:1 (1967), Albuquerque e Barretto ${ }^{1}$ (1968), Funayama e Barretto ${ }^{1 i}$ (1969), Ribeiro 30 (1973) e Mello e Teixeira 24 (1977), sobre T. cruzi isolados de roedores. Os resultados desses trabalhos mostraram sempre que as amostras de $T$. cruzi dos roedores estudados apresentavam infecçóes leves em animais de laboratório (camundongos, ratos e cobaias). As parasitemias eram sempre baixas só raramente atingindo niveis superiores a $1.000^{\prime}$ parasitos $/ \mathrm{mm}^{3}$ entre os $18-50 \%$ dia de infecção. A letalidade, também baixa, de $0-14 \%$ a exceção de uma amostra isolada de $C$. tener que foi de $46,7 \%$. A prepatencia ficou entre 4-14 dias com uma média em torno de 7 dias.

Analisando-se os dados obtidos no presente trabalho com a cepa M226 (a qual foi caracterizada especificamente no seu isolamento original por Mello e Teixeira ${ }^{24}$, 1977), verifica-se concordância com os da- 
MELLO, D. A. et al. Alguns aspectos do comportamento de cepas silvestres de Trypanosoma oruzi em camundongos e Calomys callosus (Rodentia). Rev. Saúde públ., S. Paulo, 13:314-25. 1979.

dos apresentados na literatura referida no parágrafo acima sobre a baixa virulência das cepas isoladas de roedores. De fato, analisando-se as Tabelas 3 e 5 sobre os resultados obtidos com $C$. callosus nascidos em laboratório, verifica-se que os picos de parasitemia foram baixos, variando de 530 350 entre 25 -29 \% dia após a infecção, enquanto a letalidade foi de zero. A prepatência (Tabela 1) foj em média de 9,80 dias. As infecções nos camundongos foram ainda mais leves do que as observadas em C. callosus, pois só raramente se detectava tripomastigotas no sangue circulante embora us xenos praticados nestes animais tenham sido positivos.

No que concerne à literatura sobre $T$. cruzi isolado de marsupiais, citam-se aqui os trabalhos de Correa e Barretto ${ }^{11}$ (1964), Barretto e col.5,6 (1964, 1966), Funayama e Barretto 18 (1971) que além de se referirem a estudos de amustras por eles isoladas, trazem uma extensa revisão bibliográfica sobre o assunto.

Os resultados obtidos com amostras de $T$. cruzi de $D$. albiventris e $D$. aurita, estudadas por Barretto e col.5 (1964), indicam baixa virulência tanto para camundongos quanto para ratos jovens. Como dizem os autores "a infecção é muito leve e as vezes inaparente". Os mesmos achados foram obtidos neste trabalho com as cepas R52, R64 e R65 quando inoculadas em camundongos. Como se vê na Tabela 4, entre 43 camundongos inoculados, apenas dois infectados com a cepa $\mathrm{R} 52$ e dois com R64 apresentaram uma parasitemia patente regular, embora baixa com picos de 250 a 300 parasitos $/ 5 \mathrm{~mm}^{3}$ e 3.400 a 4.750 parasitos $/ 5 \mathrm{~mm}^{3}$, respectivamente. A letalidade variou de $20 \%, 0$ e $10 \%$ para as cepas $\mathrm{R} 52, \mathrm{R} 64$ e $\mathrm{R} 65$. Os resultados obtidos com os $C$. callosus inoculados com as três cepas de $D$. albiventris indicam que estes animais sāo mais sensiveis em adquirirem a infecção do que os camundongos. Sempre apresentaram parasitemia patente com picos de 7.632 parasitos $/ 5 \mathrm{~mm}^{3}$ (R52) no $31^{\circ}$ dia de infecção, 1.225 parasitos $/ 5 \mathrm{~mm}^{3}$ (R64) no $17^{\circ}$ dia e 1.450 parasitos $/ 5 \mathrm{~mm}^{3}$ (R65) no $37^{\circ}$ dia. A letalidade para $C$. callosus foi, no entretanto, mais baixa: 6,6\% (R52), 0 (R64) e 20\% (R65).

Em trabalhos mais recentes, Zeledon e Ponce 34 (1972) estudando amostras de $T$. cruzi isoladas dos marsupiais Philanderopossum, D. marsupialis e Marmosa alstoni, em camundongos, verificaram resultados concordantes com os aqui obtidos, i.e., infecções sempre moderadas.

Embora não tenha sido objeto de estudo no trabalho ora apresentado, vale salientar, no entretanto, que foram feitas infecções experimentais por via intracerebral em camundongos de 5 dias com a cepa R52. Estes experimentos não lograram êxito no sentido de se exarcerbar a virulência da cepa. Nesse experimento foram utilizados 17 camundongos "babies" em duas ocasiões (8,9 animais) diferentes. Os resultados obtidos com estes animais foram semelhantes aos acima descritos.

$O$ encontro de pseudocistos como mostra a Tabela 6 , contendo formas amastigotas de $T$. cruzi em fibras musculares estriadas, reconfirmou a infectividade das quatro cepas silvestres de $T$. cruzi estudadas nesta pesquisa, para $C$. callosus e camundongos. A predileção do parasitismo tecidual para musculatura cardiaca e esquelética evidencia o caráter miotrópico destas cepas. Outro 
MELLLO, D. A. et al. Alguns aspectos do comportamento de cepas silvestres de Trypanosoma cruat em camundongos e Calomys callosus (Rodentia). Rev. Saúde públ., S. Paulo, 13:314-25, 1979.

aspecto histopatológico que chamou atenção neste trabalho, foi o encontro de lesões cardíacas, digestivas e da musculatura esquelética na ausência do parasito "in situ". Este achado e o encontro de fibras musculares destruidas por células mononucleares indicam que cepas de $T$. cruzi isoladas de animais silvestres são capazes de produzir reaçōes imunes de hipersensibilidade retardada, responsável pelas lesões teciduais encontradas nos grupos infectados. Achados semelhantes aos descritos aqui foram encontrados em infecções naturais em humanos, ou experimentais em animais com cepas de origem humana (Andrade 4 , 1958 e Teixeira e col.33, 1975).

Espinoza ${ }^{13}$ (1953) estudando amostras isoladas de $D$. albiventris, inoculadas em camundongos, embora não apresente descrições detalhadas, observou "lesões características e ninhos de amastigotas".

Zeledon e Ponce $3:$ (1972), verificaram que uma amostra de $T$. cruzi isolada de $D$. marsupialis apresentava neurotropismo com invasão moderada do parasito no cérebro. A maioria dos camundongos apresentavam como sintomatologia evidente, sonolência, sugerindo encefalite.

Embora no trabalho ora apresentado nào tenha sido objeto de estudo a observação em cativeiro dos animais naturalmente infectados, os $D$. albiventris, dos quais foram isoladas as cepas de $T$. cruzi R52, R64 e R65, foram mantidos vivos no laboratório até advir a morte espontânea. A sobrevivência destes animais foi a seguinte: animal infectado com a R52 sobreviveu 3 meses; com a R64, 1 ano e 6 meses e com a R65, 1 ano e 11 meses. Nestes animais foram realizados xenos e exames de sangue à fresco em períodos regulares até a morte. Os resultados dos xenos sempre foram positivos enquanto no sangue só raramente havia parasitemia patente e com niveis muito baixos.

Sem dúvida, estas observações embora preliminares, reforçam a importância de $D$. albiventris como reservatório de $T$. cruzi, considerando que em certas circunstâncias ecológicas estes marsupiais são freqüentadores assiduos do peridomicílio e domicílio. Espinoza 13 (1953), Barretto e col.5 (1964), Barretto e col. ${ }^{\circ}$ (1966), Zeledon e col. ${ }^{35}$ (1970) e Pifano ${ }^{29}$ (1973), chamam atenção para o papel epidemiológico que espécies de marsupiais do gênero Didelphis possam ter na transmissão do $T$. cruzi.

No que se refere aos dados aqui obtidos com os experimentos realizados com $C$. callosus e camundongos chamar-se-ia atenção ao fato da primeira espécie manter uma parasitemia patente, regular e por periodos longos para as quatro cepas estudadas. Sem dúvida, considerando os problemas que existem sobre isolamentos de cepas silvestres de $T$. cruzi $\mathrm{em}$ animais habituais de laboratório, os resultados aqui obtidos para C. callosus, constituem um dado importante. Como mostraram os trabalhos de Petter e col.2s (1967), Justines e Johnson ${ }^{20}$ (1970), Mello 21,23 (1977 e 1978), C. callosus é um animal de excelente adaptação em laboratório, podendo tornar-se um modelo para diferentes experimentos (Justines e Johnson ${ }^{19}, 1969$ e Mello $\left.{ }^{22}, 1978\right)$. 
MELLO, D. A. et al. Alguns aspectos do comportamento de cepas silvestres de Trypanosoma cruzi em camundongos e Calomys callosus (Rodentia). Rev. Saúde públ., S. Paulo, 13:314-25, 1979.

\section{RSPUB9/}

MELlo, D. A. et al. [Some aspects of the behavior of wild strains of Trypanosoma cruzi in mice and Calomys callosus (Rodentia)] Rev. Saúde públ., S. Paulo, 13:314-25, 1979.

ABSTRACT: Four wild strains of T. cruzi were studied for this paper: $M 226$ isolated from Calomys callosus (Rodentia) and R52, R64, and $R 65$ from Didelphis albiventris (Marsupialia). The animals were captured in the county of Formosa in the State of Goias. trypanosome strains were studied in 40 Swiss mice and in laboratory stock of $\mathrm{C}$. callosus. The following aspects were focused: parasitemia, prepatent period, lethality, and histopathologic reactions. Results indicated that these strains have low virulence for inoculated animals. Parasitemia was always low and regular for C. callosus. In mice, however, parasites were seen only in a few instances in the peripheral blood. The prepatent periods were 9.2 to 10.2 days for $\mathrm{C}$. callosus and 12 to 48 days for mice. Lethality was $7.7 \%$ for $\mathrm{C}$. callosus and $12 \%$ for mice. The parasites were found in the tissues of the inoculated animals in the proportions of $17.2 \%$ for $\mathrm{C}$. callosus and $25 \%$ for mice. Tissue reaction in both rodent species showed that all the T. cruzi strains had strong affinity for skeletal or cardiac muscle.

UnITERMS: Trypanosoma cruzi. Animals, laboratory.

\section{REFERENCIAS BIBLIOGRAFICAS}

1. ALBUQUERQUE, R. D. R. \& BARRETTO, M. P. Estudos sobre reservatórios e vetores silvestres de Trypanosoma cruzi. Infecção natural do rato d'água Nectomys squamipes (Brants 1827) pelo T. cruzi. Rev. Inst, Med. trop. S. Paulo, 10:229-37, 1968.

2. ANDRADE, S. Caracterização de cepas de Trypanosoma cruai isoladas no recôncavo baiano (contribuição ao estudo da patologia geral da doença de Chagas em nosso meio). Rev. Pat. trop., $3: 65-121,1974$.

3. ANDRADE, S. G. Cepas de Trápanosoma cruzi e sua importancia na patologia da doença de Chagas. J. bras. Med., $33: 22-3,1976$.

4. ANDRADE, Z. A. Anatomia patológica da doença de Chagas. Rev. Goiana Med., 4:103-19, 1958.

5. BARRETTO, M. P. et al, Estudos sobre reservatórios e vectores silvestres do Trypanosoma cruzi. VII. Investigaçóes sobre a infecção natural de gambás por tripanossomos semelhantes ao $T$. cruzi. Rev. bras, Biol., 4:289-300, 1964.
6. BARRETTO, M. P. et al. Estudos sobre reservatórios e vectores silvestres do Trypanosoma cruzi. XI. Observações sobre um foco natural da tripanossomose americana no municipio de Ribeirão Preto, São Paulo, Rev. Inst. Med. trop. S. Paulo, 8:103-12, 1966.

7. BRENER, $z$. The behavior of slender and stout forms of Trypanosoma cruzi in the bloodstream of normal and immune mice. Ann. trop. Med. Parasit., $63: 215-20$, 1969.

8. BRENER, Z. Comparative studies of different strains of Trypanosoma cruzi. Ann. trop. Med. Parasit., 59:19-26, 1965.

9. BRENER, Z. Contribuicão ao estudo da terapeutica experimental da doenca de Chagas. Belo Horizonte, 1961. [Tese de livre docência - Faculdade de Medicina da UFMG]

10. BRENER, Z. \& CHIARI, E. Variações morfológicas observadas em diferentes amostras de Trypanosoma cruzi. Rev. Inst. Med. trop. S. Paulo, 5:220-4, 1963. 
MELLO, D. A. et al. Alguns aspectos do comportamento de cepas silvestres de Trypanosoma cruzi em camundongos e Calomys callosus (Rodentia). Rev. Saúde públ., S. Paulo, 13:314-25, 1979.

11. CORREA, F. M. A. \& BARRETTO, M. P. Estudos sobre reservatórios e vetores silvestres do Trypanosoma cruzi. III. Infecção natural do marsupial Marmosa agilis agilis por tripanossoma semelhante ao T. cruzi. Rev. Inst. Med. trop. S. Paulo, 6:157-66, 1964.

12. DEANE, L. M. Sobre um tripanossoma do tipo cruzi encontrado num rato silvestre. no Estado do Pará. Rer. bras. Malar., 12:87-102, 1960.

13. ESPINOZA. L. G. Algunas consideraciones sobre el comportamiento del Trypano. soma cruzi (Schozotrypanum cruzi) en el Didelphis azarae o Didelphis paraguayensis (zaringueias. zorro comun o raposa). Rev. ecuat, Hig., $10: 3-4$. 1953.

14. FERRIOLLI FILHO, F. \& BARRETTO. M. P. Estudos sobre reservatórios vetores silvestres do Trypanosoma cruzi. VI. Infecção natural do roedor Akodon arviculoides curso (Winge. 1885) por tripanossomo semelhante ao Trypanosoma cruzi, Rev. Inst. Med. trop. S. Paulo, 7:72-81, 1965.

15. FERRIOLLI FILHO, F. \& BARRETTO. M. P. Estudos sobre reservatórios e vectores silvestres do Trypanosoma cruzi. IX. Infecção natural do Rattus rattus (Linnaeus 1758) por tripanossoma semelhante ao T. cruzi. Rer. Inst. Med. trop. S. Paulo, 7:169-79. 1965.

16. FERRIOLLI FILHO. F. \& BARRETTO. M. P. Estudos sobre reservatórios e vetores silvestres do Trypanosoma cruzi. XIV. Infecção natural da preá. Cavia aperea aperea. Erxleben. 1777 por tripanossoma semelhante ao $T$. cruzi. Rev. Inst. Med. trop. S. Paulo, $8: 267-76,1966$.

17. FUNAYAMA, G. K. \& BARRETTO, M. P. Estudos sobre reservatórios e vetores do Trypanosoma cruzi. XXXIV. Infecção natural do rato, oryzomys capito laticeps (Lund, 1841) pelo $T$. cruzi. Rev. bras. Biol., 29:163-73, 1969.

18. FUNAYAMA, G. K. \& BaRRETTO, M. P. Estudos sobre reservatórios e vetores silvestres do Trypanosoma cruzi. XLVI. Infecção natural do marsupial Marmosa m. microtarsus (Wagner, 1842) pelo T. cruzi. Rev, bras, Biol., 31: $263-9,1971$.
19. JUSTINES, G. \& JOHNSON, K. M. Immune tolerance in Calomys callosus infected with Machupo Virus, Nature, 222: 1090-1, 1969 .

20. JUSTINES, G. \& JOHNSON, K. M. Observations on the laboratory breeding of the Cricetinae rodent Calomys callosus. Lab. Anim. Care, 20:57-60, 1970.

21. MELlo, D. A. Biology of Calomys callosus (Rengger, 1830) under laboratory conditions (Rodentia, Cricetinae). Rer. bras. Biol., 38:807-11, 1978.

22. MELLO, D. A. Infecção experimental de Calomys callosus (Rengger, 1830). (Cricetidae-Rođentia) a quatro espécies de parasitos. Rev. Soc. bras. Med. trop. [no prelo]

23. MELLO. D. A. Note on breeding of Catomys expulsus, Lund, 1841 (Rodentia. Cricetidae) under laboratory conditions. Rev. bras. Pesq. méd. biol., 10:107. 1977.

24. MEllo, D. A. \& TEIXeira, M. L. Nota sobre a infecção natural do Calomys expulsus, Lund, 1841 (Cricetidae-Rodentia) pelo Trypanosoma oruzi. Rev. Saúde públ., S. Paulo, 11:561-4, 1977.

25. MILES, M. A. et al. The identification by isoenzima patterns of two distinet strain groups of Trypanosoma cruzi, circulating independently in a rural area of Brazil. Trans. roy. Soc. trop. Med. Hyg., 71:217-25, 1977.

26. NUSSENZWEIG, V. \& GOBLE, F. C. Further studies on the antigenic constitution of strains of Trypanosoma (Schizotrypanum) cruzi. Exp. Parasit., $18: 224-30,1966$.

27. NUSSENZWEIG, v. et al. Differences in antigenic constitution of strains of Trypanosoma cruzi. Exp. Parasit., 14:22132. 1963 .

28. PETTER, F. et al. Un nouveau rongeur de laboratoire, le Cricetidé Calomys callosus. C. R. Acad. Sci., Paris, 265: 1974-6, 1967.

29. PIFANO, F. C. La dinamica epidemiolog1ca de la enfermedad de Chagas en el valle de los naranjos, estado de Carabobo, Venezuela. I. Contribución al estudio de los focos naturales silves- 
MELLO. D. A. et al. Alguns aspectos do comportamento de cepas silvestres de Trypanosoma cruzi $\mathrm{cm}$ camundongos e Calomys callosus (Radentia). Rev. Saúde pübl., S. Paulo, 13:314-25, 1979 .

tres del Schizotrypanum cruzi Chagas. 1909. Arch. lenez. Med. trop. 5:3-29, 1973.

30. RIBEIRO. R. D. Noros reservatórios do Trypanosoma cruzi. Rer. bras. Biol., 33:423-537. 1973.

31. SIQUeIrA. A. F. et al. Estudos sobre reservatórios e rectores silvestras do Trypanosoma cruzi. XVI. Infeção na. tural do ouriço coendou insidiosus insidiosus (Kuhl, 1820) por tripanossomo semelhante ao $T$. cruzi Ror. Inst. Mcd, trop. S. Paulo, 9:155-62. 1.67.

32. SOGAYAR. R. Infecção experimental de ratos albinos wistar com diferentes cepas de Trypanosoma cruzi Chagas 1909. Belo Horizonte. 1978. [Dissertação de Mestrado - ICB/UFMG]
33. TEIXEIRA, A. R. et al. The immunology of experimental Chagas, disease in man. IV. The production of lesions in rabbits like those of chronic Chagas' ciscase in man. Amer, J. Path., 80 : 168-80, 1975.

34. ZELEDON. R. \& PONCE, C. Neurotropism in Costa Rican strains of Trypanosoma cruzi, J. Parasit., 58:180-1. 1972.

35. ZELEDON. R. et al. Wild reservoirs of Trypanosoma cruzi with special mention of the opossum Didelphis marsupialis, and its role in the epidemiology of Chagas disease in an endemic Area of Cesta Rica. J. Parasit., 56:38, 1970.

Recebido para publicąão cm 16/04/19ris Aprovado para publicacão em 19/06/19\%9 